\title{
SELETIVIDADE E EFICÁCIA DO HERBICIDA ATRAZINE + SIMAZINE, EM PÓS-EMERGÊNCIA, NA CULTURA DO MILHO, NO CERRADO
}

\author{
Júlio P. Laca-Buendia ${ }^{1}$ e J. C. Ferreira ${ }^{2}$
}

' EPAMIG, Av. Amazonas, 115 - Sala 619 - Belo Horizonte, MG 30180-902

2 EPAMIG/FEGV ,Caixa Postal 351-Uberaba, MG 38001-970

\section{RESUMO}

O objetivo do ensaio foi avaliar a eficácia da mistura atrazine + simazine, em pós-emergência na cultura de milho (cv. BR-201), em diferentes formulações e doses. O solo foi um Latossolo Vermelho-Amarelo, textura arenosa, em Uberaba, MG. Os tratamentos testados foram: Primatop $500 \mathrm{SC}$ ( atrazine $250 \mathrm{~g} / \mathrm{l}+$ simazine $850 \mathrm{~g} / \mathrm{l}$ ) na doses de $2500,3000 \mathrm{e} 3500 \mathrm{~g} / \mathrm{ha}$ e Primatop 80PM (atrazine $400 \mathrm{~g} / 1$ + simazine $400 \mathrm{~g} / \mathrm{l}$ ) nas doses de 2400, 3200 e $3600 \mathrm{~g} / \mathrm{ha}$, adicionados de óleo mineral a $0,25 \%$ $\mathrm{v} / \mathrm{v}$; Triamex SC (atrazine $250 \mathrm{~g} / \mathrm{l}+$ simazine $250 \mathrm{~g} / \mathrm{l}$ ), na dose de $3000 \mathrm{~kg} / \mathrm{ha}$; testemunha capinada e testemunha sem capina. Na densidade de plantas final, número de espigas por parcela e altura da primeira espiga, não houve diferenças significativas entre os tratamentos testados. Na produtividade de grãos, houve diferenças estatísticas entre os tratamentos estudados. A maior produção obtida foi de $3512 \mathrm{~kg} /$ ha quando se utilizou Primatop 80 PM a $3200 \mathrm{~g} /$ ha e Triamex. Na avaliação do peso de 100 sementes, o menor resultado foi obtido com Primatop 80 PM a $3500 \mathrm{~g} / \mathrm{ha}$. Para altura de planta, os herbicidas testados não apresentaram diferenças estatísticas com a testemunha capinada. Na avaliação visual para colheita mecânica, os maiores valores foram dos herbicidas Primatop 500 SC a 3000 e 3500 g/ha e Primatop 80 PM a 3200 e 3600 g/ha e Triamex. Para o controle de capimcolchão (Digitaria horizontalis) os melhores índices foram obtidos com Primatop 80 PM a 3600 g/ha e Primatop 500 SC a 3500 $\mathrm{g} / \mathrm{ha}$, com 98,5\% e 98,0\% de controle, sendo que Primatop 500 SC e Primatop 80 PM, apresentaram excelente índice de controle em todas as doses testadas. Para o controle de capim-pé-de-galinha (Eleuzine indica) os melhores índices foram encontrados com a aplicação de Primatop 80 PM a $3600 \mathrm{~kg} / \mathrm{ha}$, com 99,5\%, Primatop 80 PM a $3200 \mathrm{~g} / \mathrm{ha}$ + óleo mineral e Primatop $500 \mathrm{SC}$ a 3500 $\mathrm{g} /$ ha, com 98,5 e 98,0\%, respectivamente. Para o controle da poaia (Borreria alata) e do carrapichinho (Acanthospermum australe) Primatop 500 SC e Primatop 80 PM apresentaram um excelente controle em todas as doses testadas, até 65 dias após a aplicação. Este herbicida, nas doses e formulações testadas, não apresentou efeito de fitointoxicação nas plantas do milho cv. BR-201.

Palavras-chave: mistura de herbicida, plantas daninhas, Zea mays.

\author{
ABSTRACT \\ Efficacy of atrazine + simazine in post-emergence control of weeds in maize, \\ in Brazilian Cerrados
}

A trial was carried out to test the efficacy of formulations and doses of atrazine + simazine, in post-emergence on maize (cv. BR-201). The following treatments were tested in a sandy Latosoil, in Uberaba, Brazil: Primatop 500 SC (atrazine 250g/1+ simazine $850 \mathrm{~g} / 1$ ) at the doses of 2500,3000 and $3500 \mathrm{~g} / \mathrm{ha}$ and Primatop $80 \mathrm{PM}$ (atrazine $400 \mathrm{~g} / 1+$ simazine $400 \mathrm{~g} / 1$ ) at the doses of 2400,3200 and $3600 \mathrm{~g} / \mathrm{ha}$, plus mineral oil $(0.25 \% \mathrm{v} / \mathrm{v})$; Triamex SC (atrazine $250 \mathrm{~g} / \mathrm{l}+$ simazine $250 \mathrm{~g} / \mathrm{l})$ at the dose of $3000 \mathrm{~g} / \mathrm{ha}$; hand weeding and no control. There was no difference between treatments concerning the plant density, number of cobs and height of the first cob. The highest yield $(3512 \mathrm{~kg} / \mathrm{ha}$ ) was observed with the application of Primatop $80 \mathrm{PM}$ at $3200 \mathrm{~g} / \mathrm{ha}$ and Triamex. The lowest seed weight was observed with Primatop $80 \mathrm{PM}$ at $3500 \mathrm{~g} / \mathrm{ha}$. There was no difference between the herbicides and hand weeding concerning plant height. The best index for mechanical harvesting $(98.0-98.5 \%)$ was achieved 
with Primatop 500 SC at 3000 and $3500 \mathrm{~g} / \mathrm{ha}$, Primatop $80 \mathrm{PM}$ at 3200 and $3600 \mathrm{~g} / \mathrm{ha}$ and Triamex. The best control of Digitaria horizontalis was achieved with Primatop $80 \mathrm{PM}$ at $3600 \mathrm{~g} / \mathrm{ha}(98.5 \%)$ and Primatop $500 \mathrm{SC}$ at $3500 \mathrm{~g} / \mathrm{ha}(98.0 \%)$, but the other doses of these formulations also showed excellent control of this species. The best control of Eleusine indica was obtained with Primatop $80 \mathrm{PM}$ at $3600 \mathrm{~g} / \mathrm{ha}$ (99.5\%), Primatop $80 \mathrm{PM}$ at $3200 \mathrm{~g} / \mathrm{ha}+$ mineral oil (98.5\%) and Primatop $500 \mathrm{SC}$ at $3500 \mathrm{~g} / \mathrm{ha}$ (98.0\%). Primatop 500 SC and Primatop 80 PM showed an excellent control of Borreria alata and Acanthospermum australe at 65 days after application, in all tested doses. No symptom of fitotoxicity was observed on maize after herbicide application.

Key-words: herbicide mixture, weeds, Zea mays.

\section{INTRODUÇÃO}

O milho, no Brasil, pode ser considerada a cultura mais extensamente cultivada, principalmente em função das condições favoráveis ao seu desenvolvimento.

O período de convivência com as plantas daninhas na cultura do milho, vai desde a emergência até 45 a 50 dias após a mesma (Ruchheim Filho \& Venturella, 1977). No entanto , a cultura não deve sofrer interferência de plantas daninhas até o pendoamento (Silva \& Silva, 1987). Para a cultura do milho, perdas devido à competição com as plantas daninhas, variam de 12 a 85\% (Cruz \& Ramalho, 1983). Já Almeida (1981), no Paraná, encontrou reduções na produção de 87 a 100\%. Em São Paulo, verificaram-se reduções de $70 \%$ nas lavouras de milho não capinadas (Blanco et al.,1976).

Para o controle das plantas daninhas na cultura do milho, as triazinas tem sido estudadas por vários pesquisadores (Chehata et al., 1984; Netto et al., 1986; Almeida et al., 1988; Velini et al., 1993 e Alcântara et al., 1993).

O objetivo do ensaio de campo foi avaliar a eficácia da mistura de atrazine $250 \mathrm{~g} / 1+$ simazine $250 \mathrm{~g} / \mathrm{l}$ (Primatop $500 \mathrm{SC}$ ) + óleo mineral e atrazine $400 \mathrm{~g} / \mathrm{l}$ + simazine $400 \mathrm{~g} / \mathrm{l}$ (Primatop 80 PM) + óleo mineral, no controle de plantas daninhas através da aplicação em pós-emergência da cultura de milho, em áreas de Cerrado.

\section{MATERIAL E MÉTODOS}

O ensaio de campo foi instalado na Fazenda da Escola Agrotécnica Federal de Uberaba, MG, no ano agrícola 1993/ 94, em solo de Cerrado do tipo Latossolo Vermelho-Amarelo, textura arenosa, com $78 \%$ de areia, 16\% de argila, $6 \%$ de limo, contendo 1,2\% de matéria orgânica, $\mathrm{pH}$ (água) $=6,2$ e $68 \%$ de saturação de bases da CTC a pH=7.

A cultivar usada foi o híbrido BR-201 (Unimilho), semeado em 18/12/93, com tratamento de semente, utilizando captan $75 \%$ na dose de $1,0 \mathrm{~g} / \mathrm{kg}+$ pirimiphos-methil $50 \%$ na dose de $0,008 \mathrm{ml} / \mathrm{kg}$ de semente, no espaçamento de $1,0 \mathrm{~m}$ entre fileiras, deixando cair 6 a 7 sementes por metro linear a uma profundidade de 4 a $5 \mathrm{~cm}$, com auxilio de plantadeira mecanizada de quatro linhas.

A adubação de plantio usada foi de $350 \mathrm{~kg} / \mathrm{ha}$ da fórmula 4-30-16 de NPK $+0,38 \%$ de zinco, e na adubação nitrogenada de cobertura foram usados $100 \mathrm{~kg} / \mathrm{ha}$ com a fórmula 20-5-20 de NPK, aos 40 dias após emergência. Os tratamentos utilizados no ensaio estão discriminados na Tabela 1.

Tabela 1. Tratamentos utilizados em pós emergência, no ensaio. Uberaba-MG, 1993/94.

\begin{tabular}{lcc}
\hline \multirow{2}{*}{ Tratamento } & \multicolumn{2}{c}{ Dose } \\
\cline { 2 - 3 } & i. a. (g/ha) & p.c. $(\mathbf{k g}$ ou I/ha) \\
\hline 1-Primatop 500 SC+Assist & $2500+0,25 \% \mathrm{v} / \mathrm{v}$ & $5,0+0,25 \% \mathrm{v} / \mathrm{v}$ \\
2-Primatop 500 SC+Assist & $3000+0,25 \% \mathrm{v} / \mathrm{v}$ & $6,0+0,25 \% \mathrm{v} / \mathrm{v}$ \\
3-Primatop 500 SC+Assist & $3500+0,25 \% \mathrm{v} / \mathrm{v}$ & $7,0+0,25 \% \mathrm{v} / \mathrm{v}$ \\
4-Primatop 80PM+Assist & $2400+0,25 \% \mathrm{v} / \mathrm{v}$ & $3,0+0,25 \% \mathrm{v} / \mathrm{v}$ \\
5-Primatop 80PM+Assist & $3200+0,25 \% \mathrm{v} / \mathrm{v}$ & $4,0+0,25 \% \mathrm{v} / \mathrm{v}$ \\
6-Primatop 80PM+Assist & $3600+0,25 \% \mathrm{v} / \mathrm{v}$ & $4,5+0,25 \% \mathrm{v} / \mathrm{v}$ \\
7-Triamex 500 SC & 3000 & $6,0+0,25 \% \mathrm{v} / \mathrm{v}$ \\
8-Testemunha capinada & - & - \\
9-Testemunha sem capina & - & - \\
\hline
\end{tabular}

O ensaio foi instalado em blocos casualizados, com quatro repetições, em parcelas de $4 \mathrm{~m}$ x $5 \mathrm{~m}$, resultando uma área total de $20 \mathrm{~m}^{2}$, sendo colhidas as duas fileiras centrais, constituindo uma área útil de $10 \mathrm{~m}^{2}$ por parcela.

Os herbicidas foram aplicados em pós-emergência, em 09/01/94, quando as plantas daninhas apresentavam 2 a 4 folhas. Foi utilizado um pulverizador costal pressurizado a $\mathrm{CO}_{2}$, dotado de uma barra, com quatro bicos do tipo leque 110.04 a $0,5 \mathrm{~m}$ do solo, com vazão de 320 1/ha, usando-se uma pressão constante de $2,4 \mathrm{~kg} / \mathrm{cm}^{2}$, entre $9: 15$ e10:30 horas. O tempo se apresentava nublado, com vento moderado e o solo úmido após leve chuva de $5 \mathrm{~mm}$, com temperatura do ar de $22,8^{\circ} \mathrm{C}$ e umidade relativa de $77 \%$.

Foram realizadas três capinas manuais para o controle das plantas daninhas nas testemunhas capinadas, em 22/01/ 94, 05/02/94 e 14/03/94.

A precipitação pluvial, durante o decorrer do ensaio de campo foi de 326,4 , 358,1 , 197,4 , 255,9 e 70,6 nos meses de dezembro, janeiro, fevereiro, março e abril respectivamente, totalizando $1208,4 \mathrm{~mm}$ de chuvas no período. 
Avaliações visuais de fitointoxicação das plantas do milho foram realizadas aos 15 e 30 dias após da aplicação, através da escala visual da European Weed Research Council (EWRC), em que 1 corresponde a nenhuma injúria e 9 à morte da planta.

Aos 30, 50 e 65 dias após a aplicação realizaram-se avaliações da eficácia de controle das plantas daninhas, utilizando-se a escala percentual de 0 a $100 \%$, onde $0 \%$ corresponde a nenhum controle e $100 \%$ ao controle total (excelente), para cada espécie encontrada nas parcelas dos tratamentos. Na testemunha sem capina foi realizada a contagem por espécie dentro da área útil de cada parcela, em $1 \mathrm{~m}^{2}$.

As espécies de plantas daninhas encontradas na área estudada foram: Digitaria horizontalis (capim-colchão), com 32 plantas $/ \mathrm{m}^{2}$, Eleusine indica (capim-pé-degalinha), com 13 plantas $/ \mathrm{m}^{2}$; Borreria alata (poaia), com 8 plantas $/ \mathrm{m}^{2}$ e Acanthospermum australe (carrapichinho), com 8 plantas $/ \mathrm{m}^{2}$.

Antes da colheita foi realizada uma avaliação visual para colheita mecânica segundo a escala visual: $1=$ impossível, $2=$ =alta infestação, 3 =infestação razoável, $4=$ boa colheita e $5=$ excelente (ótimo).

Quando as plantas atingiram maturação de colheita, em 23/04/94, realizou-se a contagem das plantas de milho e a pesagem dos grãos da área útil de cada parcela. A altura média das plantas, altura de inserção da primeira espiga e o número de espigas/parcela, foram tomadas de dez plantas ao acaso, dentro de cada parcela, sendo escolhidas ao acaso 100 sementes para determinar seu peso.

\section{RESULTADOS E DISCUSSÃO}

As condições locais do ensaio de campo foram ótimas tanto para o desenvolvimento da cultura de milho, como para o desenvolvimento das plantas daninhas.

$\mathrm{Na}$ densidade de plantas final, número de espigas por parcela e altura da primeira espiga, não houve diferenças significativas entre os tratamentos testados (Tabelas 2 e 3 ).

Na produtividade de grãos houve diferenças estatísticas entre os tratamentos testados. A maior produção foi obtida com a aplicação de Primatop 80 PM $3200 \mathrm{~g} /$ ha e Triamex 500 SC, com $3512 \mathrm{~kg} /$ ha em relação a testemunha sem capina (Tabela 2).

Para a altura de planta, houve diferenças significativas, em relação à testemunha sem capina, sendo que todos os herbicidas nas doses e formulações testadas, não apresentaram diferenças estatísticas com a testemunha capinada, mas diferiram da testemunha sem capina (Tabela 2).

Verificou-se que não houve diferenças entre as misturas de herbicidas nas doses testadas para o efeito da fitointoxicação nas plantas do milho, sendo que em todos os tratamentos foi dada a nota 1 (sem injúria). Estes herbicidas, nas doses testadas, apresentaram uma alta seletividade para a cultura do milho (Tabela 3).

Para o peso de 100 sementes, houve diferenças estatísticas entre os tratamentos testados, sendo que o menor peso foi obtido quando se aplicou Primatop $80 \mathrm{PM} \mathrm{a} 3200 \mathrm{~g} / \mathrm{ha}$ + óleo mineral a $0,25 \% \mathrm{v} / \mathrm{v}, \operatorname{com} 21,1 \mathrm{~g}$ e o maior peso quando se aplicou Primatop $500 \mathrm{SC}$ a $3500 \mathrm{~g} / \mathrm{ha}$, com 26,8 g (Tabela 3).

Tabela 2. Efeitos dos herbicidas Primatop 500 SC e 80 PM, aplicados em pós-emergência, sobre a cultura do milho, em área de Cerrado. Uberaba-MG, 1993/94.

\begin{tabular}{|c|c|c|c|c|c|}
\hline Tratamento & $\begin{array}{c}\text { Dose } \\
\text { (g/ha do i. a.) }\end{array}$ & $\begin{array}{l}\text { Número de } \\
\text { plantas final } \\
\left(10 \mathrm{~m}^{2}\right)\end{array}$ & $\begin{array}{l}\text { Produtividade de } \\
\text { grãos (kg/ha) }\end{array}$ & $\begin{array}{c}\text { Altura de } \\
\text { planta }(\mathrm{cm})\end{array}$ & $\begin{array}{l}\text { Altura da } 1^{a} \\
\text { espiga }(\mathrm{cm})\end{array}$ \\
\hline Primatop $500 \mathrm{SC}+$ Assist & $2500+0,25 \% \mathrm{v} / \mathrm{v}$ & 37,2 & $3012 a b^{(1)}$ & $176,4 \mathrm{a}$ & 71,7 \\
\hline Primatop $500 \mathrm{SC}+$ Assist & $3000+0,25 \% \mathrm{v} / \mathrm{v}$ & 38,8 & $3200 \mathrm{ab}$ & $183,6 \mathrm{a}$ & 71,9 \\
\hline Primatop $500 \mathrm{SC}+$ Assist & $3500+0,25 \% \mathrm{v} / \mathrm{v}$ & 40,0 & $3450 \mathrm{ab}$ & $184,3 \mathrm{a}$ & 72,7 \\
\hline Primatop $80 \mathrm{PM}+$ Assist & $2400+0,25 \% \mathrm{v} / \mathrm{v}$ & 38,2 & $3325 \mathrm{ab}$ & $185,8 \mathrm{a}$ & 73,7 \\
\hline Primatop 80 PM + Assist & $3200+0,25 \% \mathrm{v} / \mathrm{v}$ & 41,0 & $3512 \mathrm{a}$ & $176,3 \mathrm{a}$ & 72,8 \\
\hline Primatop $80 \mathrm{PM}+$ Assist & $3600+0,25 \% \mathrm{v} / \mathrm{v}$ & 40,0 & $3125 \mathrm{ab}$ & $178,6 a$ & 73,0 \\
\hline Triamex $500 \mathrm{SC}$ & 3000 & 37,2 & $3512 a$ & $187,2 \mathrm{a}$ & 69,9 \\
\hline Testemunha capinada & - & 41,8 & $3275 \mathrm{ab}$ & $181,2 \mathrm{a}$ & 71,1 \\
\hline Testemunha sem capina & - & 42,5 & $2250 \mathrm{~b}$ & $156,2 b$ & 67,8 \\
\hline Média Geral & & 39,6 & 3185 & 178,8 & 71,6 \\
\hline Valor de F & & $0,44 \mathrm{~ns}$ & $2,36^{*}$ & $6,07 * *$ & $0,64 \mathrm{~ns}$ \\
\hline DMS & & - & 1222 & 18,2 & -- \\
\hline C.V.(\%) & & 14,49 & 15,95 & 4,25 & 6,38 \\
\hline
\end{tabular}

'As médias na mesma coluna, seguidas pela mesma letra, não diferem estatisticamente entre si, pelo teste de Tukey a $5 \%$ de probabilidade i.a. = ingrediente ativo 
Júlio P. Laca-Buendia

Tabela 3. Efeitos dos herbicidas Primatop 500 SC e 80 PM aplicados em pós-emergência, sobre a cultura do milho, em área de Cerrado. Uberaba-MG, $1993 / 94$.

\begin{tabular}{|c|c|c|c|c|c|c|}
\hline \multirow[t]{2}{*}{ Tratamento } & \multirow{2}{*}{$\begin{array}{c}\text { Dose } \\
\text { (g/ha do i. a.) }\end{array}$} & \multirow{2}{*}{$\begin{array}{c}\text { Número de } \\
\text { espigas/ } \\
\text { parcela }\end{array}$} & \multirow{2}{*}{$\begin{array}{l}\text { Peso de } 100 \\
\text { sementes (g) }\end{array}$} & \multirow{2}{*}{$\begin{array}{l}\text { Colheita } \\
\text { mecânica } \\
(1 \text { a } 5)\end{array}$} & \multicolumn{2}{|c|}{$\begin{array}{c}\text { Fitointoxicação } \\
\text { (EWRC } 1 \text { a 9) }\end{array}$} \\
\hline & & & & & 15 DAT & 30 DAT \\
\hline Primatop $500 \mathrm{SC}+$ Assist & $2500+0,25 \% \mathrm{v} / \mathrm{v}$ & 35,2 & $23,9 a b^{(1)}$ & $3,9 b$ & 1,0 & 1,0 \\
\hline Primatop $500 \mathrm{SC}+$ Assist & $3000+0,25 \% \mathrm{v} / \mathrm{v}$ & 34,8 & $24,8 \mathrm{ab}$ & $4,8 \mathrm{a}$ & 1,0 & 1,0 \\
\hline Primatop $500 \mathrm{SC}+$ Assist & $3500+0,25 \% \mathrm{v} / \mathrm{v}$ & 35,2 & $26,8 \mathrm{a}$ & $5,0 \mathrm{a}$ & 1,0 & 1,0 \\
\hline Primatop $80 \mathrm{PM}+$ Assist & $2400+0,25 \% \mathrm{v} / \mathrm{v}$ & 34,0 & $24,4 a b$ & $4,0 \mathrm{~b}$ & 1,0 & 1,0 \\
\hline Primatop 80 PM + Assist & $3200+0,25 \% \mathrm{v} / \mathrm{v}$ & 38,5 & $21,1 b$ & $4,9 \mathrm{a}$ & 1,0 & 1,0 \\
\hline Primatop 80 PM + Assist & $3600+0,25 \% \mathrm{v} / \mathrm{v}$ & 37,2 & $22,8 \mathrm{ab}$ & $5,0 \mathrm{a}$ & 1,0 & 1,0 \\
\hline Triamex 500SC & 3000 & 36,2 & $23,9 \mathrm{ab}$ & $4,8 \mathrm{a}$ & 1,0 & 1,0 \\
\hline Testemunha capinada & - & 36,5 & $23,1 \mathrm{ab}$ & $5,0 \mathrm{a}$ & 1,0 & 1,0 \\
\hline Testemunha sem capina & - & 33,2 & $20,2 b$ & $1,0 \mathrm{c}$ & 1,0 & 1,0 \\
\hline Média Geral & & 35,7 & 23,4 & 4,2 & 1,0 & 1,0 \\
\hline Valor de F & & $0,64 \mathrm{~ns}$ & $3,55^{* *}$ & $217,53 * *$ & & \\
\hline DMS & & - & 4,9 & 0,4 & & \\
\hline C.V.(\%) & & 11,46 & 8,83 & 4,12 & & \\
\hline
\end{tabular}

${ }^{1}$ As médias na mesma coluna, seguidas pela mesma letra, não diferem estatisticamente entre si, pelo teste de Tukey a $5 \%$ de probabilidade. i.a. $=$ ingrediente ativo, DAT $=$ dias após tratamento.

Tabela 4. Efeito dos herbicidas Primatop 500 SC e 80 PM, aplicados em pós-emergência, no controle de capimcolchão, na cultura do milho, no Cerrado. Uberaba-MG, 1993/94.

\begin{tabular}{|c|c|c|c|c|}
\hline \multirow{3}{*}{ Tratamento } & \multirow{3}{*}{$\begin{array}{c}\text { Dose } \\
\text { (g/ha do i. a.) }\end{array}$} & \multicolumn{3}{|c|}{ Controle $(\%)^{1}$} \\
\hline & & \multicolumn{3}{|c|}{ Dias Após os Tratamentos } \\
\hline & & 30 & 50 & 65 \\
\hline Primatop $500 \mathrm{SC}+$ Assist & $2500+0,25 \% \mathrm{v} / \mathrm{v}$ & $95,0 \mathrm{abc} c^{(2)}$ & $90,0 \mathrm{ab}$ & $87,5 b$ \\
\hline Primatop $500 \mathrm{SC}+$ Assist & $3000+0,25 \% \mathrm{v} / \mathrm{v}$ & $98,8 \mathrm{ab}$ & $98,5 \mathrm{a}$ & $98,5 \mathrm{a}$ \\
\hline Primatop $500 \mathrm{SC}+$ Assist & $3500+0,25 \% \mathrm{v} / \mathrm{v}$ & $98,8 \mathrm{ab}$ & $98,8 \mathrm{a}$ & $98,8 \mathrm{a}$ \\
\hline Primatop $80 \mathrm{PM}+$ Assist & $2400+0,25 \% \mathrm{v} / \mathrm{v}$ & $90,5 \mathrm{bc}$ & $90,0 \mathrm{~b}$ & $90,0 \mathrm{~b}$ \\
\hline Primatop $80 \mathrm{PM}+$ Assist & $3200+0,25 \% \mathrm{v} / \mathrm{v}$ & $95,0 \mathrm{abc}$ & $92,5 \mathrm{ab}$ & $92,5 b$ \\
\hline Primatop 80 PM + Assist & $3600+0,25 \% \mathrm{v} / \mathrm{v}$ & $97,5 \mathrm{ab}$ & $96,3 \mathrm{ab}$ & $93,4 b$ \\
\hline Triamex $500 \mathrm{SC}$ & 3000 & $91,5 \mathrm{c}$ & $90,0 \mathrm{~b}$ & $87,5 b$ \\
\hline Testemunha capinada & --- & $100,0 \mathrm{a}$ & $100,0 \mathrm{a}$ & $100,0 \mathrm{a}$ \\
\hline Testemunha sem capina & --- & $0,0 \mathrm{~d}$ & $0,0 \mathrm{c}$ & $0,0 \mathrm{c}$ \\
\hline $\mathrm{N}^{0}$ de plantas $/ \mathrm{m}^{2}$ & & 32,8 & 25 & 24 \\
\hline Valor de F & & $133,2 * *$ & $103,3 * *$ & $262,99 * *$ \\
\hline C.V. $(\%)$ & & 6,6 & 7,5 & 4,8 \\
\hline
\end{tabular}

(1) Dados transformados em Arco seno $\sqrt{\ldots}$ para efetuar a análise da variância.

(2) As médias na mesma coluna, seguidas pela mesma letra, não diferem estatisticamente entre si, pelo teste de Tukey a $5 \%$ de probabilidade

$\mathrm{Na}$ avaliação visual para colheita mecânica, houve diferença estatística entre os herbicidas testados, sendo que os maiores valores (entre 4,8 e 5,0) foram conseguidos pela aplicação de Primatop 500 SC a 3000 e 3500 g/ha, Primatop 80 PM a 3200 e $3600 \mathrm{~g} /$ ha e Triamex $500 \mathrm{SC}$ (Tabela 3).

Para o controle de capim-colchão, os melhores índices foram alcançados quando se usou Primatop 80 PM a $3600 \mathrm{~g} /$ ha e Primatop 500 SC a 3500 g/ha, com 98,5 e 98,0\% de controle, sendo que Primatop 500 SC e Primatop 80 apresentaram excelente índice de controle em todas as doses testadas. Para o controle de capim-pé-de-galinha, os melhores índices foram encontrados com a aplicação de Primatop 80 PM a $3600 \mathrm{~kg} / \mathrm{ha}$, com $99,5 \%$, Primatop 80 PM a 3200 g/ha e Primatop $500 \mathrm{SC}$ a $3500 \mathrm{~g} / \mathrm{ha}$, com 98 e $99 \%$, respectivamente. Para o controle da poaia e do carrapichinho, verificou-se que as aplicações de 
Tabela 5. Efeito dos herbicidas Primatop 500 SC e 80 PM, aplicados em pós-emergência, no controle de capim pé-degalinha, na cultura do milho, no Cerrado. Uberaba-MG, 1993/94.

\begin{tabular}{|c|c|c|c|c|}
\hline \multirow{2}{*}{ Tratamento } & \multirow{2}{*}{$\begin{array}{c}\text { Dose } \\
\text { (g/ha do i. a.) }\end{array}$} & \multicolumn{3}{|c|}{ Controle $(\%)^{1}$} \\
\hline & & 30 DAT & 50 DAT & 65 DAT \\
\hline Primatop $500 \mathrm{SC}+$ Assist & $2500+0,25 \% \mathrm{v} / \mathrm{v}$ & $90,0 \mathrm{~d}^{(2)}$ & $90,0 \mathrm{~d}$ & $90,0 \mathrm{~d}$ \\
\hline Primatop $500 \mathrm{SC}+$ Assist & $3000+0,25 \% \mathrm{v} / \mathrm{v}$ & $95,0 \mathrm{bc}$ & $98,0 \mathrm{ab}$ & $95,0 \mathrm{c}$ \\
\hline Primatop $500 \mathrm{SC}+$ Assist & $3500+0,25 \% \mathrm{v} / \mathrm{v}$ & $98,0 \mathrm{bc}$ & $98,5 \mathrm{ab}$ & $98,5 b$ \\
\hline Primatop $80 \mathrm{PM}+$ Assist & $2400+0,25 \% \mathrm{v} / \mathrm{v}$ & $95,0 \mathrm{~cd}$ & $95,0 \mathrm{~cd}$ & $90,0 \mathrm{~d}$ \\
\hline Primatop 80 PM + Assist & $3200+0,25 \% \mathrm{v} / \mathrm{v}$ & $97,5 \mathrm{ab}$ & $97,5 \mathrm{bc}$ & $98,5 b$ \\
\hline Primatop 80 PM + Assist & $3600+0,25 \% \mathrm{v} / \mathrm{v}$ & $99,5 \mathrm{ab}$ & $99,5 \mathrm{abc}$ & $99,5 b$ \\
\hline Triamex $500 \mathrm{SC}$ & 3000 & $94,5 \mathrm{dc}$ & $95,0 \mathrm{~cd}$ & $90,0 \mathrm{~d}$ \\
\hline Testemunha capinada & -- & $100,0 \mathrm{a}$ & $100,0 \mathrm{a}$ & $100,0 \mathrm{a}$ \\
\hline Testemunha sem capina & -- & $0,0 \mathrm{e}$ & $0,0 \mathrm{e}$ & $0,0 \mathrm{e}$ \\
\hline $\mathrm{N}^{0}$ de plantas $/ \mathrm{m} 2$ & & 14 & 13 & 16 \\
\hline Valor de F & & $401,38^{* *}$ & $291,53 * *$ & $564,96 * *$ \\
\hline C.V.(\%) & & 3,8 & 4,8 & 3,2 \\
\hline
\end{tabular}

(1) Dados transformados em Arco seno $\sqrt{x / 100}$ para efetuar a análise da variância.

(2) As médias na mesma coluna, seguidas pela mesma letra, não diferem estatisticamente entre si, pelo teste de Tukey ao nível de $5 \%$ de probabilidade. $\mathrm{DAT}=$ dias após tratamento.

Tabela 6. Efeito dos herbicidas Primatop 500 SC e 80 PM, aplicados em pós-emergência, no controle da poiaia,na cultura do milho, no Cerrado. Uberaba-MG, 1993/94.

\begin{tabular}{|c|c|c|c|c|}
\hline \multirow{2}{*}{ Tratamento } & \multirow{2}{*}{$\begin{array}{c}\text { Dose } \\
\text { (g/ha do i. a.) }\end{array}$} & \multicolumn{3}{|c|}{ Controle $(\%)^{1}$} \\
\hline & & 30 DAT & $50 \mathrm{DAT}$ & $65 \mathrm{DAT}$ \\
\hline Primatop $500 \mathrm{SC}+$ Assist & $2500+0,25 \% \mathrm{v} / \mathrm{v}$ & $90,0 c^{(2)}$ & $87,5 \mathrm{~d}$ & $87,5 \mathrm{e}$ \\
\hline Primatop $500 \mathrm{SC}+$ Assist & $3000+0,25 \% \mathrm{v} / \mathrm{v}$ & $95,0 \mathrm{bc}$ & $95,0 \mathrm{~cd}$ & $95,0 \mathrm{bcd}$ \\
\hline Primatop $500 \mathrm{SC}+$ Assist & $3500+0,25 \% \mathrm{v} / \mathrm{v}$ & $95,0 \mathrm{bc}$ & $98,0 \mathrm{~b}$ & $98,5 b$ \\
\hline Primatop $80 \mathrm{PM}+$ Assist & $2400+0,25 \% \mathrm{v} / \mathrm{v}$ & $90,0 \mathrm{c}$ & $90,0 \mathrm{~cd}$ & 90,0 cde \\
\hline Primatop $80 \mathrm{PM}+$ Assist & $3200+0,25 \% \mathrm{v} / \mathrm{v}$ & $95,0 \mathrm{bc}$ & $95,0 \mathrm{bc}$ & $95,0 \mathrm{bc}$ \\
\hline Primatop $80 \mathrm{PM}+$ Assist & $3600+0,25 \% \mathrm{v} / \mathrm{v}$ & $98,0 \mathrm{~b}$ & $98,5 b$ & $98,5 \mathrm{~b}$ \\
\hline Triamex $500 \mathrm{SC}$ & 3000 & $90,0 \mathrm{c}$ & $90,0 \mathrm{~cd}$ & $90,0 \mathrm{de}$ \\
\hline Testemunha capinada & - & $100,0 \mathrm{a}$ & $100,0 \mathrm{a}$ & $100,0 \mathrm{a}$ \\
\hline Testemunha sem capina & - & $0,0 \mathrm{~d}$ & $0,0 \mathrm{e}$ & $0,0 \mathrm{f}$ \\
\hline $\mathrm{N}^{0}$ de indivíduos $/ \mathrm{m}^{2}$ & & 8 & 9 & 15 \\
\hline Valor de F & & $352,47 * *$ & $389,73 * *$ & $467,64 * *$ \\
\hline C.V.(\%) & & 4,1 & 3,9 & 3,6 \\
\hline
\end{tabular}

(1) Dados transformados em Arco seno $\sqrt{\ldots}$ para efetuar a análise da variância

(2) As médias na mesma coluna, seguidas pela mesma letra, não diferem estatisticamente entre si, pelo teste de Tukey ao nível de $5 \%$ de probabilidade. $\mathrm{DAT}=$ dias após tratamento.

Primatop 500 SC e Primatop 80 PM, apresentaram um excelente controle em todas as doses testadas, até 65 dias após a aplicação (Tabelas 4, 5, 6 e 7).

\section{CONCLUSÕES}

1. Primatop 500 SC, nas doses de 2500, 3000 e 3500 g/ha + óleo mineral a $0,25 \% \mathrm{v} / \mathrm{v}$, demonstrou eficácia no controle de
Digitaria horizontalis (capim-colchão), Eleusine indica (capim-pé-de-galinha), Borreria alata (poaia) e Acanthospermum australe (carrapichinho), e reúne condições de praticabilidade de uso na cultura do milho, em pós-emergência das plantas daninhas mencionadas ( 2 a 4 folhas).

2. Primatop 80 PM (2400, 3200 e 3600g /ha ) + óleo mineral a $0,25 \% \mathrm{v} / \mathrm{v}$, demonstrou eficácia no controle de 
Tabela 7. Efeito dos herbicidas Primatop 500 SC e 80 PM, aplicados em pós-emergência, no controle do carrapichinho, na cultura do milho, no Cerrado. Uberaba-MG, 1993/94.

\begin{tabular}{|c|c|c|c|c|}
\hline \multirow{2}{*}{ Tratamento } & \multirow{2}{*}{$\begin{array}{c}\text { Dose } \\
\text { (g/ha do i. a.) }\end{array}$} & \multicolumn{3}{|c|}{ Controle $(\%)^{1}$} \\
\hline & & 30 DAT & 50 DAT & $65 \mathrm{DAT}$ \\
\hline Primatop $500 \mathrm{SC}+$ Assist & $2500+0,25 \% \mathrm{v} / \mathrm{v}$ & $90,0 c^{(2)}$ & $88,5 \mathrm{~d}$ & $87,5 c$ \\
\hline Primatop $500 \mathrm{SC}+$ Assist & $3000+0,25 \% \mathrm{v} / \mathrm{v}$ & $95,0 \mathrm{bc}$ & $90,5 \mathrm{~cd}$ & $90,0 \mathrm{c}$ \\
\hline Primatop $500 \mathrm{SC}+$ Assist & $3500+0,25 \% \mathrm{v} / \mathrm{v}$ & $98,0 \mathrm{~b}$ & $95,5 b c$ & $95,0 \mathrm{~b}$ \\
\hline Primatop 80 PM + Assist & $2400+0,25 \% \mathrm{v} / \mathrm{v}$ & $95,0 \mathrm{bc}$ & $90,0 \mathrm{~d}$ & $90,0 \mathrm{c}$ \\
\hline Primatop $80 \mathrm{PM}+$ Assist & $3200+0,25 \% \mathrm{v} / \mathrm{v}$ & $95,0 \mathrm{bc}$ & $98,0 \mathrm{~b}$ & $98,0 \mathrm{~b}$ \\
\hline Primatop 80 PM + Assist & $3600+0,25 \% \mathrm{v} / \mathrm{v}$ & $98,0 \mathrm{~b}$ & $98,5 b$ & $98,5 b$ \\
\hline Triamex $500 \mathrm{SC}$ & 3000 & $95,0 \mathrm{bc}$ & $90,0 \mathrm{~d}$ & $90,0 \mathrm{c}$ \\
\hline Testemunha capinada & - & $100,0 \mathrm{a}$ & $100,0 \mathrm{a}$ & $100,0 \mathrm{a}$ \\
\hline Testemunha sem capina & - & $0,0 \mathrm{~d}$ & $0,0 \mathrm{e}$ & $0,0 \mathrm{~d}$ \\
\hline $\mathrm{N}^{0}$ de individuos $/ \mathrm{m}^{2}$ & & 8 & 15 & 17 \\
\hline Valor de F & & $446,42 * *$ & $4980,09 * *$ & $557,58 * *$ \\
\hline C.V.(\%) & & 3,6 & 3,5 & 3,2 \\
\hline
\end{tabular}

(1) Dados transformados em Arco seno $\sqrt{x / 100}$ para efetuar a análise da variância.

(2) As médias na mesma coluna, seguidas pela mesma letra, não diferem estatisticamente entre si, pelo teste de Tukey ao nível de $5 \%$ de probabilidade. $\mathrm{DAT}=$ dias após tratamento.

Digitaria horizontalis (capim-colchão), Eleusine indica (capim-pé-de-galinha), Borreria alata (poaia) e Acanthospermum australe (carrapichinho), e reúne condições de praticabilidade de uso na cultura do milho, em pós-emergência das plantas daninhas mencionadas ( 2 a 4 folhas).

3. Primatop $500 \mathrm{SC}$ e Primatop 80 PM, nas doses testadas,são seletivos para a cultura do milho, híbrido BR 201, com 4 folhas, na aplicação de pós-emergência inicial.

\section{LITERATURA CITADA}

ALCÂNTARA, E. N.; SOUZA, I. F.; ALVES, A. D. Uso de triazinas em mistura com óleo mineral para o controle de plantas daninhas em milho. In: CONGRESSO BRASILEIRO DE HERBICIDAS E PLANTAS DANINHAS, 19., 1993, Londrina. Resumos dos trabalhos técnico-científicos... Londrina: SBHED, 1993, p. 142-143.

ALMEIDA, F. S. Plantio direto no Estado do Paraná Eficácia de herbicidas pós-emergentes no controle de plantas daninhas na cultura do milho. Londrina, Iapar, 1981, p. 101-144 (Circular, 23).

ALMEIDA, F. S.; FORNAROLLI, D. A.; CHEATA, A. N.; BRAZ, B. A.; BARROS, L. Controle de plantas daninhas e fitotoxicidade de diferentes misturas de atrazine, simazine, alachlor, trifluralin e diuron, na cultura do milho (Zea mays L.). In: CONGRESSO BRASILEIRO DE HERBICIDAS E PLANTAS DANINHAS, 17., Piracicaba, SP., 1988. Resumos... Ribeirão Preto: Legis Summa, 1988, p. 252-253.

BLANCO, H. G.; ARAUJO, J. B. M.; OLIVEIRA, D. A. Estudo sobre a competição das plantas daninhas na cultura do milho (Zea mays L..), determinação do período da competição. Arquivos do Instituto Biológico, São Paulo, v. 43, n. 3/4, p. 105-114, 1976.
CHEATA, A. N.; MARCONDES, D. A. S.; FORNAROLLI, D A.; BARROS, L. Efeito de altas doses de atrazine e simazine sobre a cultura do milho (Zea mays L.). In: CONGRESSO BRASILEIRO DE HERBICIDAS E PLANTAS DANINHAS, 15., e CONGRESO DE LA ASOCIACIÓN LATINOAMERICANA DE MALEZAS, 8., Belo Horizonte, MG. 1984. Resumos... Campinas: SBHED, 1984, p. $65-$ 66.

CRUZ, J. C.; RAMALHO, M. A. P. Tração animal no controle de plantas daninhas na cultura do milho. In: EMBRESA BRASILEIRA DE PESQUISA AGROPECUÁRIA-CNPMS, Sete Lagoas, MG. Mecanização na cultura do milho, utilização da tração animal. Sete Lagoas, 1983. p. 25-42 (Circular Técnica, 09).

NETTO, D. B. J.; BRAZ, B. A.; CHEATA, A. N.; HASEGAWA, $\mathrm{H}$. Comportamento da mistura pronta de atrazine+ simazine comparada com outros herbicidas utilizados na cultura do mitho (Zea mays L.). In: CONGRESSO NACIONAL DE MILHO E SORGO, 16., Belo Horizonte, MG, 1986. Resumos... Sete Lagoas: EMBRAPA/CNPMS/EMATER/EPAMIG, 1986. p. 435-445.

RUCHEIN FILHO, O.; VENTURELLA, L.R.C. Controle às plantas daninhas do milho. IPAGRO. Informe, Porto Alegre, $\mathrm{n}$. 17 , p. $45-46,1977$

SILVA, J. B da; SILVA, A. F da. Controle bem para colher melhor. Sinal Verde, São Paulo, SP. CNDA, v. 2, n. 4, p.12-13, 1987.

VELINI, E. D.; FREDERICO, L. A.; BICUDO, S. J.; ANTUNIASSI, U. R. Eficiência de herbicidas pré e pós-emergentes iniciais recomendados para a cultura do milho no controle de plantas daninhas e avaliação de efeitos dos mesmos sobre a produtividade da cultura. In: CONGRESSO BRASILEIRO DE HERBICIDAS E PLANTAS DANINHAS, 19., 1993, Londrina. Resumos dos trabalhos técnico-científicos... Londrina: SBHED, 1993, p. 141-142. 